\title{
gु
}

\section{Relaxation Decoupling in Metallic Glasses at Low Temperatures}

\author{
P. Luo, ${ }^{1}$ P. Wen, ${ }^{1}$ H. Y. Bai, ${ }^{1}$ B. Ruta, ${ }^{2,3}$ and W. H. Wang ${ }^{1, *}$ \\ ${ }^{1}$ Institute of Physics, Chinese Academy of Sciences, Beijing 100190, China \\ ${ }^{2}$ Institute of Light and Matter, UMR5306 Université Lyon 1-CNRS, Université de Lyon, 69622 Villeurbanne Cedex, France \\ ${ }^{3}$ ESRF-The European Synchrotron, CS 40220, 38043 Grenoble Cedex 9, France
}

(Received 23 November 2016; published 30 May 2017)

\begin{abstract}
Upon cooling, glass-forming liquids experience a dynamic decoupling in the fast $\beta$ and slow $\alpha$ process, which has greatly influenced glass physics. By exploring an extremely wide temporal and temperature range, we find a surprising gradual change of the relaxation profile from a single-step to a two-step decay upon cooling in various metallic glasses. This behavior implies a decoupling of the relaxation in two different processes in a glass state: a faster one likely related to the anomalous stress-dominated microscopic dynamics, and a slower one associated with subdiffusive motion at larger scales with a broader distribution of relaxation times.
\end{abstract}

DOI: 10.1103/PhysRevLett.118.225901

The relaxation dynamics of glasses is one of the most challenging unsolved problems in condensed matter physics and material science [1-5]. As the temperature is decreased below the melting point, supercooled liquids exhibit a two-step decay in the temporal evolution of the density fluctuations $[1,2]$. This behavior can be attributed to the occurrence of two distinct relaxation processes: the initial fast process is associated with rattling of the particles in the cages made by the nearest neighboring particles and escaping from the cages ( $\beta$ process), while the following slower process ( $\alpha$ process) concerns long-range translational motion after their escape from the cages [1]. In the frequency domain, the same phenomenology leads to the emergence of a broad bump at high frequency associated with fast $\beta$ relaxation, and a more pronounced peak at lower frequencies due to the slow $\alpha$ process [6,7]. Upon further cooling, the system eventually becomes a glass [3].

The relaxations in a glassy state are extremely complicated and differ remarkably from that of their high-temperature precursors supercooled liquids [8-14]. The structural rearrangements far below the glass transition temperature $T_{g}$ have been recently reported in metallic glasses (MGs) $[8,9]$ and silicate glasses $[10,11,14]$. During the aging of polymers at relatively low temperatures, the enthalpy recovery was found to experience a peculiar double-step evolution towards equilibrium, indicating the presence of different time scales for glass equilibration [12]. These findings have drawn a broad interest to the issues of glass relaxation, but meanwhile leave open the question of what is the underlying origin for such complex dynamics. MGs are considered as model systems for studying the relaxation dynamics in the glassy state, as they have the simple atomic structure and can be regarded as dense random packing of hard spheres [15]. Their aging was recently found to display distinct relaxation behaviors [14] leading even to intermittent dynamics [13].
In this Letter, we investigate the relaxation dynamics of various MGs by following the stress decay under a constant strain at temperatures ranging from $T_{g}$ down to the deep glass and time window spanning more than five decades. We find the surprising emerging of two distinct relaxation processes as the temperature decreases. While the second process corresponds to the structural $\alpha$ relaxation, the first one differs in both time scales and activation energy from the known $\beta$ relaxation, implying the occurrence of a new process proper of the glassy state that shares many similarities with the microscopic anomalous dynamics of MGs [11,14]. These results suggest the existence of additional active processes in the glassy state, which are not taken into account in previous experimental and theoretical works $[1,6]$.

Figure 1 shows the stress relaxation profiles of three typical MGs, $\mathrm{Zr}_{44} \mathrm{Ti}_{11} \mathrm{Cu}_{10} \mathrm{Ni}_{10} \mathrm{Be}_{25}\left(T_{g}=621 \mathrm{~K}\right)$, $\mathrm{Zr}_{50} \mathrm{Cu}_{40} \mathrm{Al}_{10} \quad\left(T_{g}=693 \mathrm{~K}\right)$, and $\mathrm{La}_{55} \mathrm{Ni}_{20} \mathrm{Al}_{25} \quad\left(T_{q}=\right.$ $471 \mathrm{~K})$ at different temperatures, $T$. The stress $\sigma(t)$ is normalized by its initial value $\sigma(0)$ at $t=0$. More technical details about the sample preparation and experiments can be seen in the Supplemental Material [16]. The samples are preannealed at $0.9 T_{g}$ for $48 \mathrm{~h}$ before the measurements. All systems show a decoupling of the relaxation into two steps. At temperatures around $T_{g}$, the stress $\sigma(t)$ decays in a single-step fashion (closed symbols). This behavior changes when $T$ is decreased $20-30 \mathrm{~K}$ below $T_{g}$ (open symbols). There, we do observe the formation of a shoulder for intermediate times ( $\sim 30 \mathrm{~min})$ which becomes more and more prominent upon cooling, resulting in an unexpectedly well-defined two-step decay in the deep glass.

The relaxation spectra observed here, phenomenologically bear a striking resemblance to that usually observed in the density fluctuations in Lennard-Jones glasses [2,17], molecular supercooled liquids [18], and colloidal suspensions [19-21]. In these works the fast process is independent on both $T$ and time, and is due to microscopic 

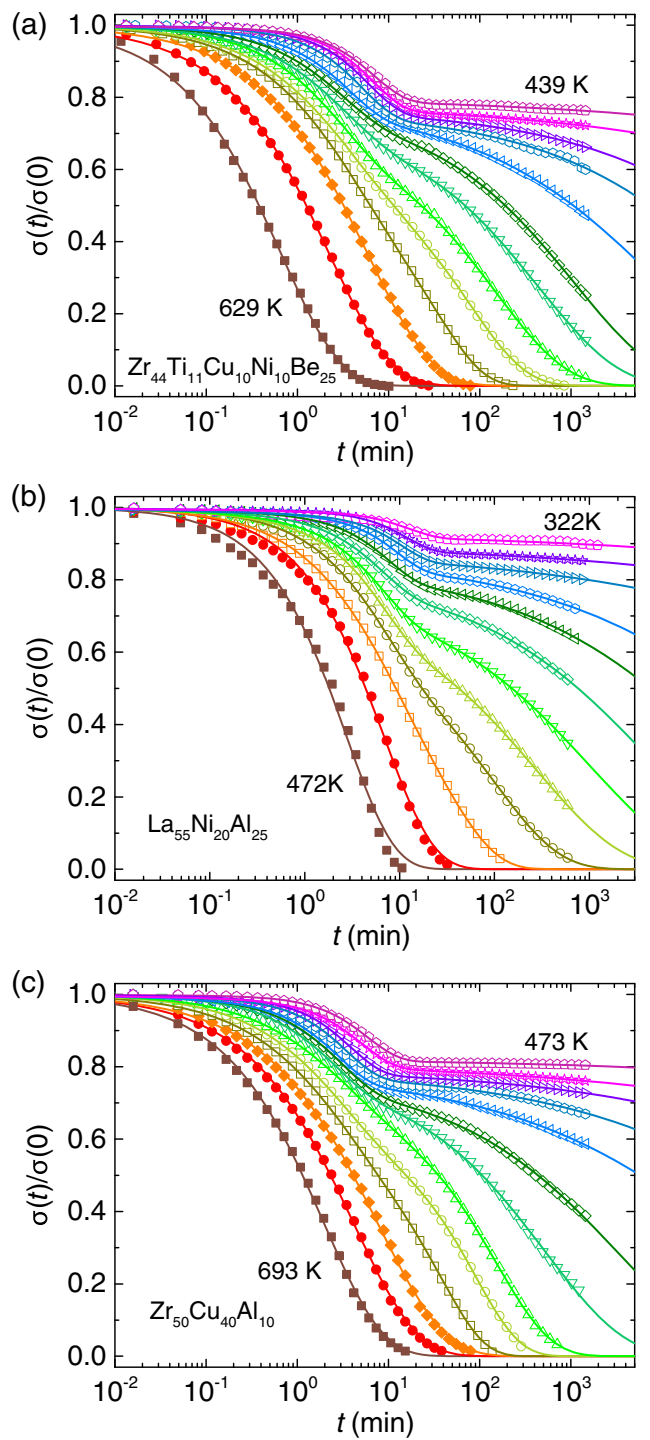

FIG. 1. (a) Stress relaxation profiles of $\mathrm{Zr}_{44} \mathrm{Ti}_{11} \mathrm{Cu}_{10} \mathrm{Ni}_{10} \mathrm{Be}_{25}$ MG, from bottom to top and left to right, $T=629,619,609,599$, 589, 579, 569, 559, 539, 519, 499, 469, and $439 \mathrm{~K}$. (b) $\mathrm{La}_{55} \mathrm{Ni}_{20} \mathrm{Al}_{25} \mathrm{MG}, T=472,462,452,442,432,422,412$, $402,382,362,342$, and $322 \mathrm{~K}$. (c) $\mathrm{Zr}_{50} \mathrm{Cu}_{40} \mathrm{Al}_{10} \mathrm{MG}, T=693$, 683, 673, 663, 653, 643, 623, 603, 583, 563, 533, 503, and $473 \mathrm{~K}$. In the stress relaxation experiments, a tensile strain $\varepsilon=0.3 \%$ is applied on the MG ribbons. Solid lines are theoretical fits to the data. The obtained fitting parameters are plotted in Fig. 2.

interactions between a particle and the cage created by its nearest neighbors. This fast process reflects a diffusive particle motion and it is usually described by a single exponential decay (thus with a shape exponent $=1$ ) [21]. In contrast with those works, here we probe the stress response in the deep nonequilibrium glassy state, and we observe a completely different additional decoupling between two dynamical processes.

At high $T$ (closed symbols in Fig. 1), the standard Kohlrausch-Williams-Watts (KWW) function,

$$
\sigma(t) / \sigma(0)=\exp \left[-\left(\Gamma_{0} t\right)^{\gamma_{0}}\right]
$$

provides an excellent fit to the data. Here, $\Gamma_{0}$ is the relaxation rate, $\gamma_{0}$ the exponent, and $t$ is the time. For lower $T$ (open symbols in Fig. 1), the model function (1) fails to describe the data and the two-step decay can be perfectly captured by a double KWW expression:

$$
\begin{aligned}
\sigma(t) / \sigma(0)= & A \exp \left[-\left(\Gamma_{1} t\right)^{\gamma_{1}}\right] \\
& +(1-A) \exp \left[-\left(\Gamma_{2} t\right)^{\gamma_{2}}\right]\left(\Gamma_{1}>\Gamma_{2}\right),
\end{aligned}
$$

where $\Gamma_{1}$ and $\Gamma_{2}$ represent the characteristic fast and slow relaxation rates, respectively, $\gamma_{1}$ and $\gamma_{2}$ are the corresponding exponents, and $A$ and (1-A) give the relaxation strength.

The parameters obtained from the fits are shown in Fig. 2 as a function of $T_{g} / T$ for better comparison. In all MGs, the relaxation rates display a universal decoupling between the fast and the slow process at $T_{g} / T \sim 1.03$ [Fig. 2(a)]. Both $\Gamma_{1}$ (half-filled symbols) and $\Gamma_{2}$ (open symbols) decrease with temperature but in a very distinct way. $\Gamma_{2}$ decreases for as large as ten decades, while $\Gamma_{1}$ varies within less than one decade over the probed temperature range. In addition, all $\Gamma_{2}$ collapse well onto a single master curve, implying the
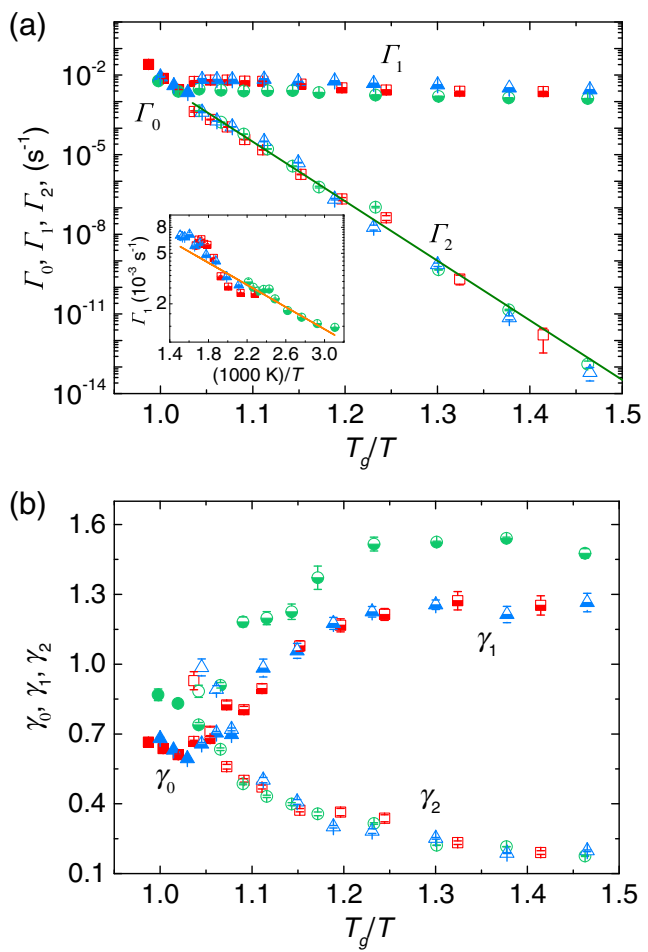

FIG. 2. The fitted relaxation rate (a) and exponent (b) as a function of $T_{g} / T$. Filled symbols represent $\Gamma_{0}$ and $\gamma_{0}$, half-filled symbols $\Gamma_{1}$ and $\gamma_{1}$, and open symbols $\Gamma_{2}$ and $\gamma_{2}$; red squares refer to $\mathrm{Zr}_{44} \mathrm{Ti}_{11} \mathrm{Cu}_{10} \mathrm{Ni}_{10} \mathrm{Be}_{25}$, green circles $\mathrm{La}_{55} \mathrm{Ni}_{20} \mathrm{Al}_{25}$, and blue triangles $\mathrm{Zr}_{50} \mathrm{Cu}_{40} \mathrm{Al}_{10} \mathrm{MG}$. The inset in panel (a) plots $\Gamma_{1}$ of the three samples against $(1000 \mathrm{~K}) / \mathrm{T}$. Error bars are given by the fitting, where they are not shown they are within the symbol size. Solid lines in panel (a) and the inset are the best linear fit. 
existence of a similar dynamics for the slowest process. The Arrhenius fit to the data yields an activation energy $\Delta E_{2}=(51.7 \pm 1.1) k_{B} T_{g}$, i.e., $2-3 \mathrm{eV}$ for MGs investigated here. This collapse does not occur for $\Gamma_{1}$ when plotted against $T_{g} / T$ (see Fig. S1 [16] for clarity), while the data overlap well when reported as a function of the inverse temperature $(1000 \mathrm{~K}) / T$ [inset in Fig. 2(a)]. Here, the Arrhenius fit yields an activation energy $\Delta E_{1} \sim 0.1 \mathrm{eV}$ independent of $T_{g}$, or rather, of the composition.

According to our current knowledge, two main kinetic mechanisms are responsible for relaxation in the glassy state, i.e., the fast $\beta$ Johari Goldstein (JG) relaxation and the slow structural $\alpha$ process [6,7]. At first sight, it seems plausible that the unexpected fast decay observed here can be ascribed to the JG relaxation. However, these two processes are incompatible with each other on both the time scale and the apparent activation energy. In MGs, the relaxation rate of the $\beta$ process is typically in the order of $1 \mathrm{~s}^{-1}$ around $0.7 T_{g}$ (see, e.g., Refs. [6,7]), while our process is much slower, with a rate of already $\sim 10^{-3} \mathrm{~s}^{-1}$ at the first step [see Fig. 2(a)]. In addition, the apparent activation energy of $\beta$ relaxation depends on $T_{g}$ and it would be approximately $26 k_{B} T_{g}$ for MGs [6,22], i.e., 1.1-1.6 eV for our systems. As mentioned before, remarkably, the fast decay reported here has almost negligible apparent activation energy $\sim 0.1 \mathrm{eV}$, which strongly contrasts with the usual $\beta$ relaxation, and cannot therefore be ascribed to this well-known process. The initial decay herein shows different timescales and activation energy with respect to those observed in enthalpy relaxation of glassy polymers [12]. This could be related to the different nature of the systems and the probed temperature range. Indeed, the data in Ref. [12] correspond to $T_{g} / T$ in the range of 1-1.05, thus to a narrow range where our two processes do still merge [Fig. 2(a)], and the first process exhibits relatively low activation energy seemingly decreasing with temperature. Notwithstanding, in both cases, the occurrence of an additional step during the glass relaxation implies a complex dynamic behavior during structural relaxation not accounted for in previous macroscopic studies [15], which leads to a reconsideration of many phenomenological models for relaxation in glasses $[1,23]$.

Figure 2(b) shows the temperature dependence of the KWW exponents. At high temperature $\sigma(t)$ exhibits a unique stretched decay $\left(\gamma_{0}<1\right)$, where $\gamma_{0}$ decreases with temperature. In the two-step regime the data can be described by two KWW decays with distinct exponents: as $T$ decreases the exponent of the first decay, $\gamma_{1}$ (half-filled symbols), increases progressively from $\sim 0.7$ to above 1 , finally saturating at $\sim 1.3$ for both $\mathrm{Zr}$-based MGs and at $\sim 1.5$ for the La-based MG. Conversely, the exponent of the second decay $\gamma_{2}$ is less than 1 , continuously decreases with $T$, and is independent on glass composition. This contrasting going-up versus going-down evolution of $\gamma_{1}$ and $\gamma_{2}$ with cooling highlights the distinct nature beyond the two relaxation processes. The peculiar behavior that $\gamma_{1}$ reaches above 1 for $T_{g} / T>1.1$ is in clear contrast with the following slower step and with previous works [3-5] where the stress response has been always associated with a stretched exponential decay, with thus a shape parameter lower than 1. Such anomalous compressed $(\gamma>1)$ decay has been observed in the microscopic dynamics of a variety of systems, including soft matters [24-31], MGs $[13,14,32,33]$, and even magnetic structures [34], and it has been described in terms of a ballistic particle motion relaxing randomly distributed internal local stress dipoles [24-26]. Differently, the long time dynamics can be associated with the typical slower subdiffusive motion and heterogeneous dynamical scenario $[5,25]$. The dominant role of internal stresses in the fast process is also confirmed by the low activation energy of $\Gamma_{1}$ (less than $0.1 \mathrm{eV}$ ) which suggests an almost negligible contribution from thermal energy. A similar phenomenon has been seen also in density fluctuations of a colloidal suspensions close to the jamming transition [31]. Here the KWW exponent increases from below to above 1 and finally saturates around 1.3 as the volume fraction increases [31]. In MGs the density increases by approximately $1.5 \%$ from $\sim T_{g}$ to room temperature [35]. Thus, it seems plausible that the increase of $\gamma_{1}$ is associated with the proximity of the glass transition and the densification of the system as temperature decreases. This is consistent with the picture of the local stress dipoles dominated ballisticlike particle motion preceding the long-range structural rearrangements of the whole matrix.

To shed some light on the mechanism responsible for the observed occurrence of distinct relaxation processes in the deep glassy state, we studied this phenomenon as a function of the annealing time and for different strains. Figure 3(a) shows the stress relaxation profiles of $\mathrm{La}_{55} \mathrm{Ni}_{20} \mathrm{Al}_{25}$ glasses with different ages, i.e., as-cast and preannealed at $0.9 T_{g}$ for different annealing times $t_{a}$. The as-cast sample shows a normal fast nonexponential decay $\left(\gamma_{0} \sim 0.7\right)$. As the sample ages, the decay splits in two step. We find that the relaxation rate of the slow process decreases with $t_{a}$ as a power law $\Gamma_{2} \sim t_{a}^{-2 / 3}$, whereas the first process appears independent on $t_{a}$ [see the inset in Fig. 3(a)]. In both cases, the shape of the curves remains basically constant, with $\gamma_{1} \sim 1.2$ and $\gamma_{2} \sim 0.5$. Figure 3(b) shows the response function of $\mathrm{Zr}_{44} \mathrm{Ti}_{11} \mathrm{Cu}_{10} \mathrm{Ni}_{10} \mathrm{Be}_{25}$ under different strains at $519 \mathrm{~K}$, the samples are preannealed at $0.9 T_{g}$ for $48 \mathrm{~h}$. The upper right inset in Fig. 3(b) shows the initial stress before relaxation as a function of the imposed strain. As the strain reaches $\sim 1 \%$, the stress deviates from the linear dependence on strain, i.e., yielding starts. At smaller strains (i.e., below $0.7 \%$ ), the normalized relaxation strength associated with the fast process increases with strain. In the plastic flowing regime (above 1\%) the relaxation still follows a two-step behavior and the curves collapse on the top of each other. Curiously, the corresponding relaxation 

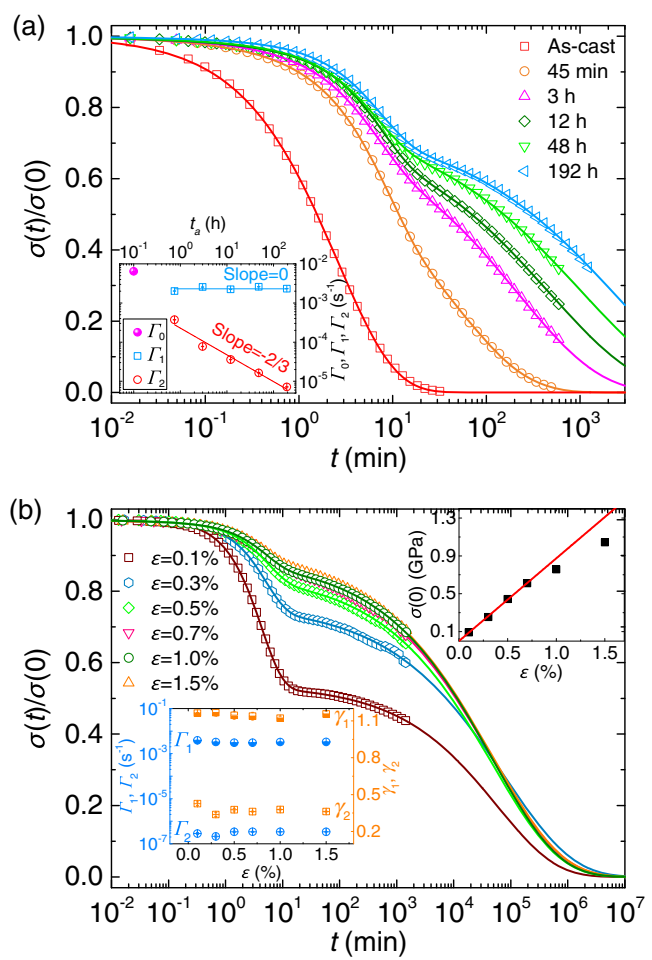

FIG. 3. (a) Stress relaxation profiles of $\mathrm{La}_{55} \mathrm{Ni}_{20} \mathrm{Al}_{25} \mathrm{MG}$ of different ages: as-cast and pre-annealed at $0.9 T_{g}(422 \mathrm{~K})$ for a series of planned times, $t_{a} \cdot \varepsilon=0.3 \%$ and $T=422 \mathrm{~K}$. The data for the as-cast sample are fitted with Eq. (1) and the others Eq. (2). The inset shows the relaxation rate versus $t_{a}$, where the data for the as-cast sample is also plotted in the logarithmic coordinates at $t_{a}=0.1 \mathrm{~h}$. (b) Stress relaxation profiles of $\mathrm{Zr}_{44} \mathrm{Ti}_{11} \mathrm{Cu}_{10} \mathrm{Ni}_{10} \mathrm{Be}_{25} \mathrm{MG}$ at $519 \mathrm{~K}$ with different strains, fitted with Eq. (2). The lower left and upper right insets plot the relaxation rate and exponent, and $\sigma(0)$ versus strain, respectively.

rates and shape exponents appear independent of the imposed strain within the experimental uncertainty [see the lower left inset in Fig. 3(b)]. Such a two-step behavior provides, thus, a new clue to understand the plastic deformation of MGs which deserves additional studies.

From the viewpoint of the potential energy landscape (PEL) $[3,36,37]$, the stress decay is indicative of rearrangements in the system to release the additional nonequilibrium energy with sampling alternate local minima. During the aging, the system explores deeper energy minima with higher barriers and the frequency of transitions between local minima reduces [36]. As a consequence, the relaxation rate of the second process decreases [Fig. 3(a)]. Interestingly, the fast process is not affected by the aging and has almost negligible apparent activation energy. This suggests that during this fast process, the glass is likely trapped in an energy basin where it relaxes on few atomic distances to release local stresses prior to the slow translational motion. At high $T$ or for high energy state (like, e.g., in the as-cast sample) on the PEL, the structural relaxation occurs on faster time scales, resulting in a merging of these two processes in a single decay of the stress function. Strain causes a decrease of the height of the barriers within different minima and can lead to a curvature of the potential energy surface [37]. In this scenario different strain would result in different morphology. Our data show that the profile of the stress relaxation remains unchanged even beyond the yielding regime [Fig. 3(b)]. An intuitive understanding is that the dynamics will not be affected by the morphology change of PEL provided that the relative position of the system on the PEL still remains unchanged.

The finding of decoupling of the relaxation dynamics extends our knowledge of glasses far beyond the orthodox understanding of glass relaxation, and a more complete scenario can be sketched. As schematically shown in Fig. 4, at high $T$, the dynamics of viscous liquids can be described by the cage rattling of the particles and their escape from the cage, with the subsequent decoupling in $\beta$ and $\alpha$ relaxations. In a glassy state, we do observe the new decoupling: the faster process could be related to microscopic internal stresses annihilation, likely due to ballisticlike particle motion, while the slower mode appears associated with a broader distribution of relaxation times probably associated with larger length scales. Distinct dynamical processes and intermittent structural relaxation have been recently observed also in the atomic motion of various glasses by means of x-ray photon correlation spectroscopy (XPCS) [11,13,14,32,33]. Intriguingly, we note that the measured relaxation times occur on time scales comparable with that of the initial fast step in our data, and both processes share the analogous compressed exponential decay associated with internal stresses [13,14,32,33]. XPCS provides indirect information on density fluctuations on a microscopic scale of few $\AA[14,32]$. The absence of the second slow process in that XPCS data is likely due to its

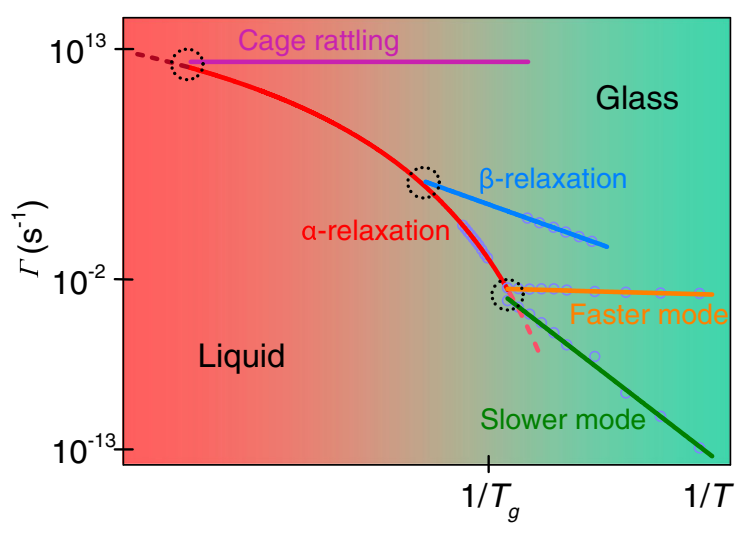

FIG. 4. Schematic Arrhenius diagram concerning dynamical behaviors of MG and its high temperature precursors. Data for $\alpha$ and the $\beta$ relaxations are from dynamical mechanical measurements on $\mathrm{La}_{55} \mathrm{Ni}_{20} \mathrm{Al}_{25} \mathrm{MG}$ (Fig. S3 [16]). The red line is the Vogel-Fulcher-Tammann (VFT) [3] fit to the $\alpha$-relaxation data and the blue line the Arrhenius fit to the $\beta$-relaxation data. 
macroscopic nature, being associated with the relaxation of the whole matrix. Our findings suggest the existence of a direct link between microscopic and macroscopic relaxations, opening the field to further studies aimed to clarify the rich dynamics and in-depth mechanisms of glass relaxation.

Recently, there have been debates to ascertain whether there exists a finite temperature divergence of the dynamics below $T_{g}$ [38-43]. Our experimental evidence shows the existence of two distinct relaxation processes in a glassy state. While the fast process has a clear nondivergent nature as it does not display any relevant temporal or temperature dependence, the second process displays a marked Arrhenius dependence on the temperature [see Fig. 2(a)], which as well is not compatible with a diverging scenario. Nevertheless, such a complex relaxation spectrum could not exclude the presence of a diverging time scale.

We are indebted to W. Kob, M. Ballauff, M. Fuchs, T. Voigtmann, Y.Z. Li, Y.T. Sun, H.L. Peng, M.Z. Li, and Y. L. Han for very helpful discussions. This research was supported by the NSF of China (51571209 and 51461165101), the MOST 973 Program (2015CB856800), and the Key Research Program of Frontier Sciences, CAS (QYZDY-SSW-JSC017).

*Corresponding author.

whw@iphy.ac.cn

[1] E. Donth, The Glass Transition (Springer, Berlin, 2001).

[2] W. Kob and H. C. Andersen, Phys. Rev. E 52, 4134 (1995).

[3] P. G. Debenedetti and F. H. Stillinger, Nature (London) 410, 259 (2001).

[4] J. C. Phillips, Rep. Prog. Phys. 59, 1133 (1996).

[5] M. D. Ediger, Annu. Rev. Phys. Chem. 51, 99 (2000).

[6] H. B. Yu, W. H. Wang, H. Y. Bai, and K. Samwer, Natl. Sci. Rev. 1, 429 (2014).

[7] P. Luo, Z. Lu, Z. G. Zhu, Y.Z. Li, H. Y. Bai, and W. H. Wang, Appl. Phys. Lett. 106, 031907 (2015).

[8] P. Luo, Z. Lu, Y.Z. Li, H. Y. Bai, P. Wen, and W. H. Wang, Phys. Rev. B 93, 104204 (2016).

[9] Z. Lu, W. Jiao, W. H. Wang, and H. Y. Bai, Phys. Rev. Lett. 113, 045501 (2014).

[10] R. C. Welch, J. R. Smith, M. Potuzak, X. Guo, B. F. Bowden, T. J. Kiczenski, D. C. Allan, E. A. King, A. J. Ellison, and J. C. Mauro, Phys. Rev. Lett. 110, 265901 (2013).

[11] B. Ruta, G. Baldi, Y. Chushkin, B. Rufflé, L. Cristofolini, A. Fontana, M. Zanatta, and F. Nazzani, Nat. Commun. 5, 3939 (2014).

[12] D. Cangialosi, V. M. Boucher, A. Alegria, and J. Colmenero, Phys. Rev. Lett. 111, 095701 (2013).

[13] Z. Evenson, B. Ruta, S. Hechler, M. Stolpe, E. Pineda, I. Gallino, and R. Busch, Phys. Rev. Lett. 115, 175701 (2015).
[14] B. Ruta, Y. Chushkin, G. Monaco, L. Cipelletti, E. Pineda, P. Bruna, V. M. Giordano, and M. Gonzalez-Silveira, Phys. Rev. Lett. 109, 165701 (2012).

[15] W. H. Wang, Prog. Mater. Sci. 57, 487 (2012).

[16] See Supplemental Material at http://link.aps.org/ supplemental/10.1103/PhysRevLett.118.225901 for the technical details about the experiments and more supplemental figures.

[17] W. Kob and J. L. Barrat, Phys. Rev. Lett. 78, 4581 (1997).

[18] N. Petzold, and E. A. Rössler, J. Chem. Phys. 133, 124512 (2010).

[19] Z. Zheng, F. Wang, and Y. Han, Phys. Rev. Lett. 107, 065702 (2011).

[20] G. Brambilla, D. El Masri, M. Pierno, L. Berthier, L. Cipelletti, G. Petekidis, and A. B. Schofield, Phys. Rev. Lett. 102, 085703 (2009).

[21] F. A. de Melo Marques, R. Angelini, E. Zaccarelli, B. Farago, B. Ruta, G. Ruocco, and B. Ruzicka, Soft Matter 11, 466 (2015).

[22] F. Zhu, H. K. Nguyen, S. X. Song, D. P. B. Aji, A. Hirata, H. Wang, K. Nakajima, and M. W. Chen, Nat. Commun. 7, 11516 (2016).

[23] L. Berthier and G. Biroli, Rev. Mod. Phys. 83, 587 (2011).

[24] L. Cipelletti, S. Manley, R. C. Ball, and D. A. Weitz, Phys. Rev. Lett. 84, 2275 (2000).

[25] J.-P. Bouchaud and E. Pitard, Eur. Phys. J. E 6, 231 (2001).

[26] E. E. Ferrero, K. Martens, and J. L. Barrat, Phys. Rev. Lett. 113, 248301 (2014).

[27] H. Guo, G. Bourret, M. K. Corbierre, S. Rucareanu, R. Bruce Lennox, K. Laaziri, L. Piche, M. Sutton, J. L. Harden, and R. L. Leheny, Phys. Rev. Lett. 102, 075702 (2009).

[28] R. Aravinda Narayanan, P. Thiyagarajan, S. Lewis, A. Bansal, L. S. Schadler, and L. B. Lurio, Phys. Rev. Lett. 97, 075505 (2006).

[29] D. Orsi, E. Guzmán, L. Liggieri, F. Ravera, B. Ruta, Y. Chushkin, T. Rimoldi, and L. Cristofolini, Sci. Rep. 5, 17930 (2015).

[30] P. Bursac, G. Lenormand, B. Fabry, M. Oliver, D. A. Weitz, V. Viasnoff, J. P. Butler, and J. J. Fredberg, Nat. Mater. 4, 557 (2005).

[31] P. Ballesta, A. Duri, and L. Cipelletti, Nat. Phys. 4, 550 (2008).

[32] V. M. Giordano and B. Ruta, Nat. Commun. 7, 10344 (2016).

[33] B. Ruta, G. Baldi, G. Monaco, and Y. Chushkin, J. Chem. Phys. 138, 054508 (2013).

[34] O. G. Shpyrko et al., Nature (London) 447, 68 (2007).

[35] A. R. Yavari, A. Le Moulec, A. Inoue, N. Nishiyama, N. Lupu, E. Matsubara, W. J. Botta, G. Vaughan, M. Di Michiel, and A. Kvick, Acta Mater. 53, 1611 (2005).

[36] A. Heuer, J. Phys. Condens. Matter 20, 373101 (2008).

[37] D. L. Malandro and D. J. Lacks, Phys. Rev. Lett. 81, 5576 (1998).

[38] T. Hecksher, A. I. Nielsen, N. B. Olsen, and J. C. Dyre, Nat. Phys. 4, 737 (2008). 
[39] J. Zhao, S. L. Simon, and G. B. McKenna, Nat. Commun. 4, 1783 (2013).

[40] V. Lubchenko and P. G. Wolynes, Annu. Rev. Phys. Chem. 58, 235 (2007).

[41] G. B. McKenna and J. Zhao, J. Non-Cryst. Solids 407, 3 (2015).
[42] D. Cangialosi, J. Phys. Condens. Matter 26, 153101 (2014).

[43] E. A. A. Pogna, C. Rodríguez-Tinoco, G. Cerullo, C. Ferrante, J. Rodríguez-Viejo, and T. Scopigno, Proc. Natl. Acad. Sci. U.S.A. 112, 2331 (2015). 